\title{
Desigualdad: el término más antónimo de la pobreza
}

\section{Inequality: the most antonym term of poverty}

Jaime Leopoldo Cabezas Maldonado

Universidad Internacional del Ecuador, Ecuador

Carlos Vásquez Villavicencio

Universidad Internacional del Ecuador, Ecuador

Autor para correspondencia: edlarcoca@uide.edu.ec, xavrod7@ hotmail.com

Fecha de recepción: 29 de Septiembre de 2017 - Fecha de aceptación: 15 de Noviembre de 2017

Resumen: La sociedad ha llegado a asimilar los conceptos de desigualdad y pobreza como sinónimos, lo que ha incidido tanto en el pensamiento político como en el económico, la creencia de que la solución para extirpar la pobreza es combatir la desigualdad. Rallo (2017) ha afirmado recientemente que: Dentro del imaginario colectivo, los conceptos de "pobreza" y de "desigualdad" han terminado por fusionarse: si hay pobres es porque somos desiguales; si la desigualdad aumenta, es porque ha aumentado la pobreza (...) lo que deja de perder (o lo que gana) uno es porque lo pierde otro. A partir de este enfoque se analiza a la desigualdad, la que ha sido totalmente mal comprendida por la filosofía política y la teoría económica a nivel mundial, al desconocer que su esencia en sí está ligada con la naturaleza humana, razón por la cual, es más bien la desigualdad la que puede brindar el ambiente propicio para la creación y descubrimientos de las oportunidades de desarrollo individual de las personas y de la economía en su conjunto. Se busca desligar a la desigualdad de las teorías equívocas que la relacionan con la pobreza, tales como las expuestas por Piketty (2014) quien ha afirmado: "Cuando la tasa de rendimiento del capital supera la tasa de crecimiento de la producción y los ingresos (...) el capitalismo genera automáticamente las desigualdades arbitrarias e insostenibles que socavan radicalmente los valores meritocráticos en que se basan las sociedades democráticas."(p. 4). El presente estudio pretende explicar cómo la desigualdad puede llevarnos a demostrar que es más bien el intervencionismo estatal, al buscar la igualdad a toda costa, el verdadero generador de la pobreza.

Palabras clave: desigualdad; pobreza; capitalismo; libre mercado

Abstract: Society has come to assimilate the concepts of inequality and poverty as synonyms, which has influenced both political and economic thinking, the belief that the solution to eradicate poverty is to combat inequality.

From this concept we analyze how inequality has been totally misunderstood by political philosophy and economic theory at the world level, by not knowing that its essence in itself is linked to human nature, which is why it is rather the inequality which can provide the environment conducive to the creation and discovery of opportunities for individual development of people and the economy as a whole. It seeks to bridge the inequality of the equivocal theories that relate it to poverty, such as those put forward by Piketty (2014). The present study aims to explain how inequality can lead us to demonstrate, which is rather the state interventionism in seeking equality at all costs, the true generator of poverty.

Key words: inequality; poverty; capitalism; free market 


\section{Introducción}

Dentro del pensamiento social actual se ha llegado a unificar y confundir dos conceptos: la desigualdad en los ingresos y la pobreza; el no separarlos ha provocado que muchos teóricos los consideren causa y consecuencia. La relevancia en el tratamiento dado a estos dos conceptos se ha transformado en el eje central de la política económica en muchos países, al encontrarse en el discurso e ideario de muchos líderes políticos y de opinión.

Este fenómeno no solo se observa en América Latina sino también en todo el mundo. Ejemplos de esto los podemos ver en la reciente conferencia del Foro Económico Mundial (WEF por sus siglas en inglés) llevado adelante en Davos Suiza. Según la Agenda Global, en la que se expusieron las cinco prioridades de liderazgo para 2017 por parte del presidente ejecutivo y fundador de esta organización, Klaus Schwab ha manifestado: que se debe tratar a la pobreza y a la desigualdad como males a ser eliminados por igual. Según él, los mercados libres y la globalización han mejorado el nivel de vida y han sacado a la gente de la pobreza durante décadas, pero sus defectos estructurales han promovido el aumento de la desigualdad en el reparto de la riqueza. (WEF, 2017). De la misma manera, el discurso de Barack Obama, unos meses antes de dejar la Casa Blanca, afirmó que la desigualdad se ha transformado en un mal y por tanto debe ser combatido, ya que según su parecer, esa sería la razón por la que existe pobreza tanto en su país como en el mundo.

El problema principal de este malentendido es que estos dos términos y su real significado, no podían estar más contrapuestas, debido a las evidencias que nos presenta la historia. Analizar de una manera profunda y sólida la diferencia esencial entre estos dos conceptos, ayudará a clarificar cómo muchas de las percepciones que inducen al error y que muchos estudiosos consideran correctas, no son sino, especulaciones alejadas de la realidad, cuya verdad es demostrable con aquellas evidencias que se encuentran por doquier en el mundo real.

\section{La desigualdad está presente desde que el ser humano existe}

En primer lugar, la desigualdad no es algo reciente, no se originó hace poco y no es culpa del mercado, de los empresarios, ni del capitalismo. La desigualdad es tan antigua y tan consustancial a la existencia de la vida y se la aprecia aún en el reino animal. Los seres más capaces y los que se esfuerzan más, buscan mejorar sus condiciones de vida y asegurar su subsistencia. A partir de esto, podemos comprender que:

Nunca ha habido ni jamás habrá una sociedad de iguales en un sentido material. Y no la habrá porque los seres humanos somos únicos, es decir, desiguales en un sentido concreto. Cuando se permite que las personas actúen con libertad, el complejo conjunto de características que nos diferencian, sumado a factores como el azar, deriva en resultados necesariamente desiguales. De este modo, la desigualdad material es, en parte, la inevitable consecuencia de la diversidad humana. Por ello el proyecto de lograr igualdad material solo puede intentarse destruyendo la libertad, conduciendo invariablemente a la tiranía y la miseria. (Kaiser, 2012, p. 1)

Desde esta perspectiva, es muy difícil pensar que la desigualdad es algo que se puede controlar. Las personas actuamos como entes económicos independientes, buscamos satisfacer 
nuestras necesidades de la mejor manera que nos es posible, buscamos realizar actividades bajo nuestras propias preferencias y por ende, el beneficio que recibimos de una actividad es muy diferente para cada una de las personas. En el pasado, estas desigualdades económicas se debían a muchos factores. Schettino (2014) ha afirmado: "Las grandes diferencias en todo ese tiempo [pasado] tenían su origen en el nacimiento y en la pertenencia a un grupo social determinado. Casta, estrato, grupo, determinaban la vida y la muerte. Y claro, también el acceso a la riqueza" (p.1).

Sin embargo, gracias al libre mercado y al sistema capitalista, en la actualidad las brechas de la pobreza se han reducido, cambiando esta realidad, no en términos de igualdad, sino en disminuir las barreras y diferencias sociales. ¿Cómo ha sido posible esto?; se ilustra con la siguiente historia:

El creador de la firma Amazon, Jeff Bezos, junto a su esposa comenzó en el garaje de una casa arrendada, un negocio de venta de libros vía internet. Gracias a sus capacidades y esfuerzo, la originalidad y brillante idea de la forma como podían comercializarse, transformó a esta pareja de esposos de escasos recursos, en dueños de una de las empresas más exitosas de la actualidad. Podemos asumir que en la época que Jeff comenzaba a idear la creación de esa empresa, las oportunidades que él y su vecino tenían eran muy parecidas, incluso tal vez era mejor la situación de su vecino, pues la casa de la familia Bezos era arrendada; sin embargo, la creatividad y las capacidades únicas de cada ser humano, que es una característica propia de la desigualdad, hicieron que los Bezos se transformen en una de las familias más ricas en la actualidad.

Esto puede ser complementado por las palabras de Adam Smith quien señaló que cada individuo ha sido creado para perseguir su propio interés a su manera, dentro de un plan de igualdad, libertad y justicia, que se genera de una manera espontánea (...) (Hayek, 1978, p.2).

\section{La desigualdad es una oportunidad y no una desventaja}

Los líderes mundiales que juegan el rol de agentes de cambio social condenan a la desigualdad, a pesar de la evidencia. Sin embargo, es ésta condición la que permite que las personas que más ahorros poseen, los pueden invertir en actividades productivas y empresariales, lo que termina mejorando el bienestar de los demás, además del suyo propio, dando como resultado el que así se puedan satisfacer las infinitas necesidades humanas y sus preferencias.

Un beneficio de la desigualdad es que podemos disfrutar los resultados de la creatividad e innovación particular de cada individuo, de su permanente e innato estado de alerta y perspicacia empresarial, por tanto lo que le corresponde a la sociedad es premiar a las personas que han invertido los recursos de su propiedad para crear nuevas formas de satisfacer las necesidades humanas entendidas como los fines infinitos que persigue cada individuo según sus preferencias. Por eso tenemos que considerar como valiosas las grandes oportunidades que nos brinda el tener una sociedad desigual, lo cual permite refutar el argumento de la desigualdad como algo injusto.

Podríamos mencionar a los deportes como otro ejemplo. Para disfrutar este tipo de espectáculos se paga un precio y al hacerlo se crea un bienestar para el espectador, mientras al mismo tiempo el producto de las recaudaciones permite el pago a los jugadores, premiando el 
ingreso de los mejores. El hecho de que Cristiano Ronaldo gane millones de dólares por temporada ¿afecta al bienestar de los espectadores? La respuesta lógica es que los afecta, pero de manera positiva, pues mejora el bienestar de los espectadores. No nos tiene por qué perturbar el enriquecimiento de un individuo cuyas capacidades innatas o adquiridas son una cuestión de cada ser humano. Quien nos brindó el servicio tiene todo el derecho de gozar de unas rentas diferenciadas, con mayor razón si el bienestar producido por ese servicio mejora las condiciones de millones de seres humanos mediante la recreación.

Esto no solo sucede en el mundo de los deportes, podemos aplicarlo a cualquier bien o servicio que consumimos. La computadora que utilizamos, el teléfono móvil inteligente, el vehículo por medio del cual nos transportamos, los libros que compramos, etc. y muchos nuevos bienes que día a día se crean y están disponibles en el mercado. Quienes los han creado y aquellas personas que día a día compiten, cooperan y coordinan para producirlos, tienen todo el derecho de apropiarse del fruto de su inventiva y creatividad, lo cual obviamente los vuelve desiguales a los demás, razón por la cual nadie tiene el derecho de reprimir o castigar este comportamiento, pues hacerlo básicamente sería inmoral e inhumano al impedir el avance de la civilización.

En un ambiente de sociedades desiguales, se promueven las oportunidades individuales, donde las personas pueden progresar y desarrollarse como entes capaces. Es importante tomar en cuenta el principio económico que nos enseña que los individuos responden a los incentivos y que cada quien persigue el fin que más valora y lo hace con los medios con los que cuenta, basado en un entorno de respeto a la propiedad privada y de libertad para actuar. Desde esta perspectiva, podemos comprender el avance en estos últimos dos siglos de los conocimientos y la tecnología, donde millares de individuos y empresas año a año remozan y mejoran la gama de productos y servicios que se ofrecen al mercado, para así beneficiarse a sí mismo y a la sociedad.

Podemos ver en el extremo contrario como sociedades y países calificados como más "iguales", como el caso de Mongolia, Nepal o Tayikistán (Banco Mundial, 2014), al limitar la libertad de empresa, han desincentivado la innovación e invención dentro de sus países, a tal grado de ser sociedades "iguales" extremadamente pobres y atrasadas. Como lo manifestó Hayek en una de sus frases más famosas: "solo existen dos posibilidades: ser muchos y ricos, o pocos y pobres".

\section{La pobreza y el capitalismo}

Algunos pensadores usando la ideología, han llegado a asociar el concepto de pobreza al de las sociedades libres, o en la visión de Marx, al capitalismo, término creado por él mismo para referirse a las sociedades del libre mercado que recién se gestaban en el siglo XIX. Es tal el problema de la asociación de estos términos que el mismo Papa Francisco, en su visita a Bolivia, hablando de un supuesto "capitalismo desenfrenado" expresó: "Este sistema ya no se aguanta, no lo aguantan los campesinos, no lo aguantan los trabajadores, no lo aguantan los pueblos. Y tampoco lo aguanta la Tierra, la hermana madre Tierra como decía San Francisco". (WEF, 2017)

Claramente, el Papa estaba omitiendo el hecho de que gracias al sistema del libre mercado, se ha logrado transformar a los pobres campesinos en trabajadores asalariados, mucho 
más ricos de lo que Carlos Marx hubiese esperado con la aplicación de su utópica propuesta constante en su obras "El manifiesto comunista" o "El Capital". Somos parte de una época de la historia humana en la cual, como nunca antes ha sucedido, se han reducido drásticamente los índices de pobreza, sacando a más de mil millones de esta situación en el último medio siglo, gracias al sistema de mercado que mayoritariamente está presente en el mundo, aunque todavía atado a muchos obstáculos que impone el intervencionismo estatal.

En tal caso, ¿por qué el Papa se expresó en su discurso en contra del capitalismo que, según él, somete y explota a Bolivia? Es algo que muchas personas no comprenden. Para entenderlo de mejor manera sobre la situación boliviana Ricardo Hausmann (2015) ha afirmado:

Las empresas más rentables del mundo no están explotando a Bolivia: simplemente, no se encuentran ahí porque consideran que el país no es rentable. El problema más fundamental del mundo en desarrollo es que el capitalismo no ha reorganizado la producción ni el empleo en los países y regiones más pobres, con lo que la mayor parte de la fuerza laboral ha quedado fuera de su ámbito operacional. (p.1)

Es importante separar a la pobreza y al capitalismo de nuestra mente. El verdadero problema es que los países pobres tienen desconfianza del sistema capitalista; muchas veces por ideología o desconocimiento y buscan deliberadamente la intervención del gobierno para regular la vida económica, provocando que el capitalismo no prospere, que la economía de mercado opere de manera disfuncional y que finalmente estos países permanezcan más bien en la pobreza.

\section{Piketty y su frustración debido a la pobreza y desigualdad}

En el año 2013, Thomas Piketty escribió El Capital en el Siglo XXI, un libro con 577 páginas de texto, 76 páginas de notas y 115 gráficos y tablas, que se ha convertido en todo un éxito en la lista de best sellers según el New York Times. Su obra se centra en la tesis de que el rendimiento del capital excede a la tasa de crecimiento económico de un país, por lo tanto propone que el Estado implemente "un impuesto global progresivo sobre el capital" para detener el crecimiento "desmedido" del patrimonio de los más ricos.

Al parecer la obra de Piketty no toma en cuenta que desde el siglo XIX y gracias al comercio y la empresarialidad, la proporción de personas que se han enriquecido es siete veces mayor que la que existía en 1800. El problema principal según este autor, es la desigualdad, a la que considera erróneamente antiética. Al igual que otros llamados progresistas de izquierda, no explica el por qué la desigualdad por sí misma es perjudicial. Tal vez en opinión de él, no es ético que alguien por su trabajo, ideas o esfuerzo tenga tres casas, cuatro autos, o use un reloj de más de diez mil dólares.

El principal problema que presenta la ética de la lucha contra la desigualdad es que busca que el Estado intervenga cada vez más para solucionar este supuesto problema de la sociedad, siendo más bien lo que parece ser la cura, una suerte de enfermedad social moderna que la podemos definir como "estatolatría". Esta solución incluye el tener un gobierno enorme, que consume una inmensa parte de la renta nacional, con todos los problemas que ese mal reparto genera, para promover el paternalismo, que busca como cuidar a los ciudadanos de la cuna a la tumba, lo que le resta el sentido de responsabilidad a los individuos respecto de su propia vida y 
a su vez instaura el proteccionismo a los pseudo-empresarios, léase buscadores de rentas, asegurándoles utilidades a costa de sobreprecios que los paga la sociedad, logros que se los alcanza controlando coactivamente la vida de las personas, para que mediante el uso de la fuerza y la violencia institucionalizada de un Estado cada vez más intervencionista, se implante una sociedad utópicamente igualitaria.

Bajo esta visión que no es nueva, se instauraron las peores tiranías en la historia de la humanidad. Tenemos el caso de la desaparecida $\operatorname{URSS}^{1}$ y los países que se los llamó tras la "cortina de hierro" como Alemania del Este, sistema que terminó en 1989 con la caída del Muro de Berlín. Desgraciadamente subsisten la actual Corea del Norte, Cuba y se han estado gestando en el mundo entero, nuevas sociedades basadas en el concepto de la igualdad, tal el caso de Venezuela y otras tiranías populistas cada vez más centralizadas y estatizadas.

Para comprender el libro de Piketty, McCloskey (2015) argumentó: La visión apocalíptica de Piketty deja poco espacio para el resto de nosotros a los que realmente nos va muy bien — de manera no apocalíptica más bien-como de hecho nos ha ido desde 1800. Lo que le preocupa a Piketty es que los ricos puede que posiblemente se vuelvan más ricos, aun cuando los pobres también se enriquecen. Su preocupación es puramente acerca de la diferencia, sobre un confuso sentimiento de envidia elevado a una propuesta teórica y ética. (p.1)

\section{Desigualdad o falta de libertad: ¿cuál de ellas explica de mejor manera la pobreza?}

Aunque el Índice de Gini ${ }^{2}$ nos ofrece información interesante sobre la cual muchos estudiosos y políticos sociales basan sus argumentos a favor de la igualdad, también nos brinda información contrapuesta sobre la realidad de la pobreza frente a la desigualdad. Por ejemplo, Suecia, un país con una sociedad indudablemente desarrollada, ocupa uno de los primeros lugares del índice (27.3), sin embargo, si solo consideramos ese factor, podríamos decir entonces que países como Bielorrusia (26.53), Kirguistán (27.3), Kazajstán (27.4) y Moldavia (29.16), deberían encontrarse dentro de las naciones más prosperas del planeta. Basados en esta escala, estos países serían más ricos que Francia (33.1), España (35.9) y Reino Unido (32.57). (Banco Mundial, 2015)

La desigualdad está muy lejos de ser la causante de la pobreza dentro de las naciones. Sin embargo, podemos encontrar una clave que puede ayudarnos a aclarar la razón de ser de la pobreza: el Índice de Libertad Económica ${ }^{3}$. Si analizamos conjuntamente este índice de libertad y lo comparamos con el coeficiente de Gini, descubriremos que existen muchas naciones, que aunque son muy igualitarias, al quitar libertades a sus habitantes, se mantienen como naciones

\footnotetext{
${ }^{1}$ Siglas de la desaparecida Unión de Repúblicas Socialistas Soviéticas

${ }^{2}$ El coeficiente de Gini es un método usado especialmente en economía para medir la desigualdad en los ingresos. El valor 0 expresa igualdad total, mientras que un valor de 1 sería la máxima desigualdad.

${ }^{3}$ El índice de Libertad Económica en el Mundo mide el grado de apoyo a la libertad económica en las políticas y las instituciones de cada país. Las piedras angulares de la libertad económica son la elección personal, el intercambio voluntario, la libre competencia y la garantía de la propiedad privada. Se utilizan 42 parámetros para elaborar un índice global que mide el grado de libertad económica en cinco grandes áreas: 1 Tamaño del Estado: gasto, impuestos y empresas; 2 Estructura jurídica y garantía de los derechos de propiedad; 3 Acceso a una moneda sana; 4 Libertad de comercio internacional y 5 Regulación crediticia, laboral y de la empresa. Se calificó en 2012 con una puntuación sobre 10, con base a la información de 152 países.
} 
muy pobres. Es tal el caso de Tayikistán, donde a pesar de tener uno de los mejores índices de igualdad según Gini (30.8), el intervencionismo estatal característico de las naciones de la antigua Unión Soviética, ha llegado a generar problemas muy grandes de inestabilidad y pobreza en dicho país.

La FAO (2017) en su informe de proyectos considera que "la malnutrición sigue siendo generalizada" dentro de Tayikistán. (p.1). Otro problema grande es la migración masiva de los habitantes hacia Rusia por la falta de empleo. Se calcula que 1.58 millones de tayikos viven en Rusia. (Sávina, 2016)

Lastimosamente, el caso de Tayikistán es solo uno de muchos más, que al final de cuentas solo nos muestran la inconsistencia de querer combatir la desigualdad con más estatización. El principal problema al que nos enfrentamos en esta temática son las propuestas políticas de combate a la pobreza, que no ofrecen una solución a este problema, sino solo un discurso retórico para tener sociedades más iguales, pero no prósperas; es decir, se aplica una ética consistente en quitar al más rico empeorando su vida, para así supuestamente ayudar al más pobre. Esta estrategia no toma en cuenta que en ninguna nación donde se la aplicó ha funcionado eficazmente. Lo único que ha logrado es acrecentar el tamaño del Estado, talvez con sociedades más igualitarias, pero también más pobres.

Con el objeto de ilustrar con datos tomados de los reportes especializados, se presenta la siguiente tabla mostrando para algunos países el coeficiente de GINI y la posición en materia de libertad que ocupan en el mundo algunos países seleccionados.

Tabla 1. Comparación entre Índice de Gini y posición en el Índice de Libertad Económica en el Mundo

\begin{tabular}{lccc}
\hline \multicolumn{1}{c}{ País } & $\begin{array}{c}\text { Índice de Gini } \\
\text { Calificación/posición entre 152 países }\end{array}$ \\
\hline Bielorrusia & 0.265 & - & - \\
Suecia & 0.273 & 7.47 & 32 \\
Kirguistán & 0.273 & 6.77 & 90 \\
Kazajstán & 0.274 & 7.00 & 74 \\
Moldavia & 0.291 & 6.85 & 86 \\
Tayikistán & 0.308 & 6.67 & 97 \\
Reino Unido & 0.325 & 7.81 & 12 \\
Francia & 0.331 & 7.21 & 58 \\
España & 0.359 & 7.29 & 51 \\
Venezuela & 0.407 & 3.89 & 152 \\
Ecuador & 0.454 & 6.01 & 131 \\
\hline
\end{tabular}

Nota. Tomado y adaptado de Banco Mundial. (2015). Gini Index (World Bank estimate). Banco de datos Indicadores del desarrollo. Para el caso de Venezuela, se tomó de la data de la Comisión Económica para América Latina CEPAL.

\section{Conclusión}

Finalmente, después de haber analizado estas posiciones, podemos darnos cuenta que la desigualdad está lejos de ser la causa de la pobreza. De hecho, el problema real de la pobreza es el intervencionismo del Estado. Políticas como cerrarse al comercio, a la globalización, a la inversión extranjera, para enfocarse en el proteccionismo, han causado que muchas naciones no puedan salir de la pobreza y se mantengan en ese nivel durante muchos años. Podemos ver esta 
realidad en el caso de Venezuela, que nos pueden servir de ejemplo para conocer qué es lo que sucede cuando en un país se eliminan sistemáticamente las libertades, se destruye la propiedad confiscando y expropiando mediante impuestos y otros mecanismos, la renta y el patrimonio de los individuos y a costa de querer volver a los ciudadanos más igualitarios a la fuerza, se provoca que sean más bien igualitariamente pobres. Se ha demostrado entonces que la desigualdad es el término más antónimo de la pobreza.

\section{Bibliografía}

Banco Mundial. (2015). Gini Index (World Bank estimate). Banco de datos Indicadores del desarrollo, Recuperado de http://databank.bancomundial.org/data/reports.aspx?source=2\&series=SI.POV.GINI\&cou ntry=\#selectedDimension_WDI_Ctry

CEPAL. (2017). Data del índice de GINI. Recuperado de http://estadisticas.cepal.org/cepalstat/WEB_CEPALSTAT/buscador.asp?idioma=e\&stri g_busqueda=gini

FAO. (2017). Tayikistán adopta una nueva vía para luchar contra la malnutrición infantil. Boletín de La Organización de las Naciones Unidas para la Alimentación y la Agricultura. Recuperado de: http://www.fao.org/ in-action/tajikistan-embraces-a-new-way-to-fight child-malnutrition/es/

Gwartney, J., Lawson, R. y Hall, J. Libertad Económica en el Mundo, Informe 2014. Recuperado de https://www.elcato.org/sites/default/files/efw14_0.pdf

Hausmann, R. (2015). ¿Es el capitalismo la causa de la pobreza?, La Nación. Recuperado de http://www.nacion.com/opinion/foros/capitalismo-causa-pobreza_0_1509049116.html

Hayek, F.A. (1978). El mensaje de Adam Smith en el lenguaje actual. (Ensayo). New Studies. Recuperado de www.hacer.org/pdf/Hayek02.pdf

Hayek, F.A. Revista del Instituto Juan de Mariana IJM Actualidad. Recuperado de https://www.juandemariana.org/ijm-actualidad/pildoras-de-libertad/fa-hayek-gigante-del orden-espontaneo

Kaiser, A. (2012). Justa desigualdad. Boletín Semanal El Cato. Recuperado de https://www.elcato.org/justa-desigualdad

McCloskey, D. (2015). Piketty ignora lo más importante. Boletín Semanal El Cato. Recuperado de: https://www.elcato.org/piketty-ignora-lo-mas-importante

Papa Francisco. (2017). ¿Es el capitalismo la causa de la pobreza? Boletín del World Economic Forum. Recuperado de https://www.weforum.org/es/agenda/2015/08/es-el-capitalismo-la causa-de-la-pobreza/

Piketty, T. (2014). EL Capital en el siglo XXI. Recuperado de https://jcguanche.files.wordpress.com/2015/02/piketty-el-capital-en-siglo-xxi-1.pdf

Rallo, J.R. (2017). Desigualdad no es Pobreza. Boletín Semanal El Cato. Recuperado de https://www.elcato.org/desigualdad-no-es-pobreza

Sávina, K. (2016). Tayikistán un país sin hombres. Russia Beyond the Headlines. Recuperado de https://es.rbth.com/pol\%C3\%ADtica-y-sociedad/sociedad/2016/10/12/tayikistan-un-pais sin-hombres_638187

Schettino, M. (2014). Desigualdad económica y desigualdad de derechos. Boletín Semanal El Cato. Recuperado de https://www.elcato.org/ desigualdad-económica-y-desigualdad-de derechos

Schwab, K. (2017). Cinco prioridades de liderazgo para 2017. Boletín del World Economic Forum. Recuperado de https://www.weforum.org/es/ agenda/2017/01/cinco-prioridades-de liderazgo-para-2017 